\title{
Explanation of Capacitive Performance of the Plasma in Damavand Tokamak
}

\author{
Shervin Goudarzi1, Fatemeh Dadgarnejad'2, Hojat Babaee1 \\ ${ }^{1}$ Atomic Energy Organization of Iran, Nuclear Science and Technology Research Center, Plasma Physics and \\ Nuclear Fusion Research School, Tehran, Iran \\ ${ }^{2}$ Nuclear Science \& Technology Research Institute, Atomic Energy Organization of Iran, Tehran, Iran \\ Email: sgoudarzi@aeoi.org.ir
}

Received 29 December 2014; accepted 15 January 2015; published 2 February 2015

Copyright (C) 2015 by authors and Scientific Research Publishing Inc.

This work is licensed under the Creative Commons Attribution International License (CC BY).

http://creativecommons.org/licenses/by/4.0/

(c) (i) Open Access

\begin{abstract}
In this work capacity of tokamak plasma is calculated using modeling of tokamak configuration as toroidal and coaxial capacitor. This value is very important and plays an important role in timevarying regimes in tokamak. For exact simulation of plasma behavior, this amount will be added to circuit equations and transport codes. Since capacitive properties of tokamak cause production of a radial electric field, it deserves our special attention.
\end{abstract}

\section{Keywords}

\section{Tokamak, Capacitive Properties, Radial Electric Field}

\section{Introduction}

Tokamak is a torodial shape magnetic confinement fusion device that is the best candidate for nuclear fusion reactors [1] [2]. Working principle of this device is like a transformer, passing the electric current through the primary coils inducing a current in the tokamak plasma that plays the role of secondary coils of transformer (Figure 1) [1]-[3]. This current causes heating of the plasma and creates a polar magnetic field $B_{\theta}$ which increases the quality of the confinement of tokamak plasmas. The resistive and inductive properties of the plasma in tokamak have been widely studied. Though the plasma has capacitance property in all area between its centre and the chamber wall, its capacitive performance was not widely studied before 1990's [4] [5]. In this article, the capacitive property of the plasma in the tokamak and its importance in circuit equations and transport codes of tokamak is explained. Then, the numerical results of such model for Damavand tokamak compare with experimental results and a good agreement between them is observed. 


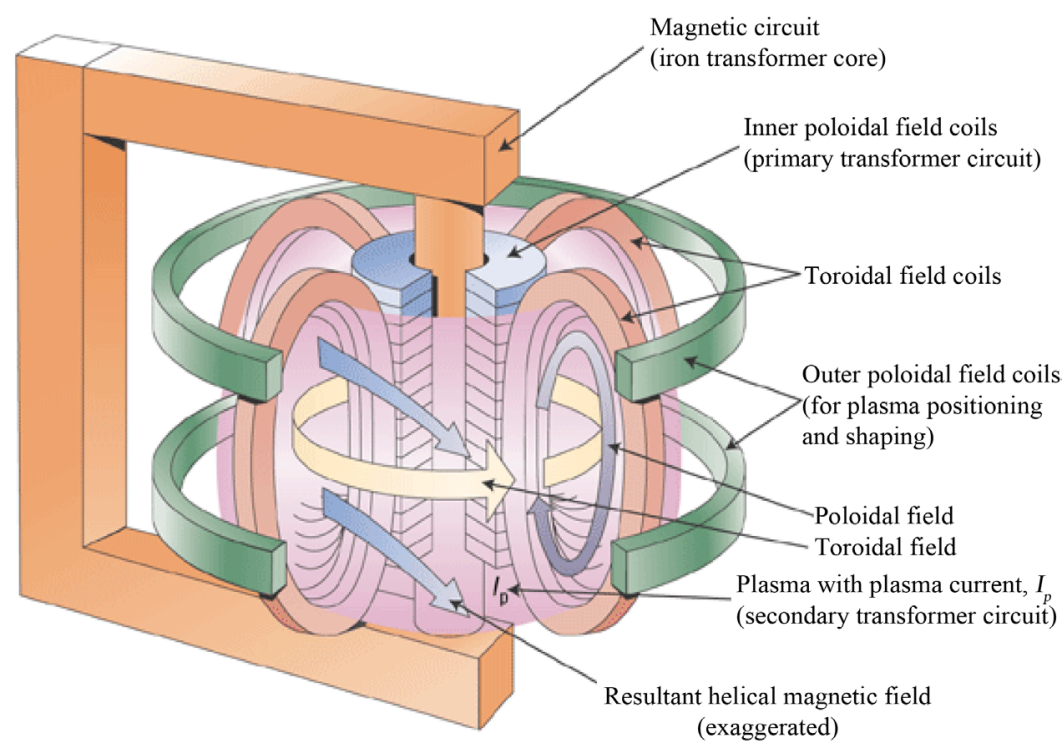

Figure 1. Tokamak [3].

\section{The Capacitive Model}

Estimation the value of capacitance of the equal circuit of tokamak can be done by a torodial coaxial capacitor (Figure 2), the tokamak plasma plays the role of inner electrode and the discharge chamber wall is external electrode and the low-density plasma between them considered as the dielectric of capacitor. In this article, the capacity of tokamak plasma obtained through solving the Laplace equation for a torus, and the working regimes in which this property is important have been identified.

$a_{1}, \quad a_{2}$ and $R$ are the distance between centre of plasma and its edge, tokamak minor radius and major radius of the tokamak, respectively. Here the main problem is the solving of the Laplace equation for $V(r, \theta, \phi)$ with the boundary conditions of ( $V=0$ in $\left.r=a_{1}\right)$ and $\left(V=1\right.$ in $\left.r=a_{2}\right)$.

The Laplace equation [6]:

$$
\begin{gathered}
\nabla^{2} V=\frac{1}{h_{1} h_{2} h_{3}}\left[\frac{\partial}{\partial r}\left(\frac{h_{2} h_{3}}{h_{1}} \frac{\partial V}{\partial r}\right)+\frac{\partial}{\partial \theta}\left(\frac{h_{1} h_{3}}{h_{2}} \frac{\partial V}{\partial \theta}\right)+\frac{\partial}{\partial \phi}\left(\frac{h_{1} h_{2}}{h_{3}} \frac{\partial V}{\partial \phi}\right)\right]=0 \\
h_{1}=1, \quad h_{2}=r \quad \text { and } h_{3}=R-r \cos \theta
\end{gathered}
$$

With substituting the above values in Equation (1) and with respect to that in this system $\frac{\partial V}{\partial \phi}=0$ the Equation (1) becomes as follows.

$$
\begin{gathered}
\frac{\partial}{\partial r}\left[r\left(1-\frac{r \cos \theta}{R}\right) \frac{\partial V}{\partial r}\right]+\frac{\partial}{\partial \theta}\left[\left(\frac{1-r \cos \theta}{R}\right) \frac{\partial V}{\partial \theta}\right]=0 \\
r=a_{1}: V=0 \\
r=a_{2}: V=1
\end{gathered}
$$

Because of the uniqueness of the solutions of Laplace equation, there will be only one answer that will satisfy the above conditions.

It is obvious that in a constant $\frac{r}{R}$, the solution of Equation (2) $V$ will be a periodic function of $\theta$ that its period is $2 \pi$. It is also evident that because of symmetry $V$ is a paired function of $\theta$. Thus, $V$ can be described as a cosine Fourier series of $\theta$ as the following form in which coefficients $\left(b_{n}\right)$ are functions of $\frac{r}{R}$ : 


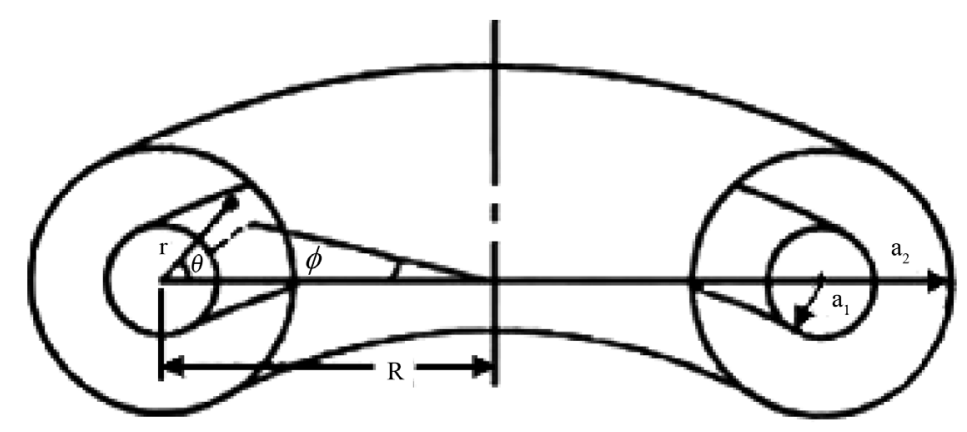

Figure 2. Coaxial torus [2].

$$
V\left(\frac{r}{R}, \theta\right)=\sum_{n=0}^{\infty} b_{n}\left(\frac{r}{R}\right) \cos (n \theta)
$$

Now, it is necessary to obtain expressions for $b_{n}\left(\frac{r}{R}\right)$ that can satisfy the boundary conditions, for finding the simplest solution, we first put the $V\left(\frac{r}{R}, \theta\right)$ from Equation (3) instead of $V$ in Equation (2) and equals the coefficients of $\cos (p \theta)$ in the resulting equation to zero for any natural number of $p$ more than 1 . Finally, the following expressions obtain for $b_{0}$ and $b_{1}$ :

$$
\begin{gathered}
b_{0}=\left(\frac{r}{R}\right)^{2}+\left\{\left[1-\left(\frac{a_{2}}{R}\right)^{2}\right] \operatorname{Ln}\left(\frac{r}{a_{1}}\right)+\left(\frac{a_{1}}{R}\right)^{2} \operatorname{Ln}\left(\frac{r}{a_{2}}\right)\right\} / \operatorname{Ln}\left(\frac{a_{2}}{a_{1}}\right) \\
b_{1}=\frac{4}{R}\left(r+\frac{a_{1} a_{2}}{r}-a_{1}-a_{2}\right)
\end{gathered}
$$

On the other hand, the solution to Laplace equation for such coordinate system would be in the form of

$$
V_{0}=\frac{\operatorname{Ln}\left(\frac{r}{a_{1}}\right)}{\operatorname{Ln}\left(\frac{a_{2}}{a_{1}}\right)}
$$

This solution provides the boundary conditions $V\left(a_{1}\right)=0$ and $V\left(a_{2}\right)=1$ and is completely independent from $\theta$. We can calculate the capacity of capacitor in Figure 2 if the charge on each torus can be measured. With respect to that the boundaries are considered as co-potential surface, on them $\frac{\partial V}{\partial \theta}=0$. Therefore, the electrical charge on each surface of torus is equal to $Q=\varepsilon \int_{s} E_{r} \mathrm{ds}$ in which the integral is done over the entire of surface.

In this relation $E_{r}=-\left(\frac{\partial V}{\partial R}\right)_{r=a_{1}}$ and $\varepsilon$ is the coefficient of permittivity of dielectric material and $\mathrm{d} s=a_{1}\left(R-a_{1} \cos \theta\right) \mathrm{d} \theta \mathrm{d} \phi$.

$$
Q=\varepsilon \int_{\varphi=0}^{2 \pi} \int_{\theta=0}^{2 \pi} a_{1}\left(R-a_{1} \cos \theta\right) \frac{\partial}{\partial r}\left[b_{0}+b_{1} \cos \theta+\sum_{n=2}^{\infty} b_{n}\left(\frac{r}{R}\right) \cos (n \theta)\right]_{r=a_{1}} \mathrm{~d} \theta \mathrm{d} \varphi
$$

By putting the calculated values for $b_{0}$ and $b_{1}$ (Equation (4)) in Equation (5)) and notice that all of the coefficients $b_{n}$ for $n \geq 2$ are equal to zero and integrating it the value of $Q$ is obtained by Equation (6):

$$
Q=-4 \pi^{2} \varepsilon R\left[\frac{1+\left(a_{1} / R\right)^{2}-\left(a_{2} / R\right)^{2}}{\operatorname{Ln}\left(a_{2} / a_{1}\right)}+\frac{2 a_{1} a_{2}}{R^{2}}\right]
$$


Since $C=\frac{|Q|}{V}$ and $V=1$, the value of capacitance can be calculated by following equation:

$$
C=4 \pi^{2} \varepsilon R\left[\frac{1+\left(a_{1} / R\right)^{2}-\left(a_{2} / R\right)^{2}}{\operatorname{Ln}\left(a_{2} / a_{1}\right)}+\frac{2 a_{1} a_{2}}{R^{2}}\right]
$$

In limit of $R \rightarrow \infty$ and taking into account $L=2 \pi R$ Equation (7) is changed to $C=\frac{2 \pi \varepsilon L}{\operatorname{Ln}\left(a_{2} / a_{1}\right)}$ which is the same formula for capacitance in concentric cylinders. It should be noted that $\varepsilon$ is the permittivity of the space between the boundary of plasma and the vacuum chamber that is a low-density plasma and can be written as $\varepsilon=\varepsilon_{0} \varepsilon_{\perp}$ that $\varepsilon_{\perp}=1+\frac{c^{2}}{V_{A}^{2}}, \quad V_{A}=\frac{B}{\left(\mu_{0} \rho\right)^{1 / 2}}$ is the Alfven velocity and $B, \rho$ and $c$ are the strength of magnetic field, mass density and velocity of light, respectively. Because the values of $\rho$ in different regions are not equal, also the values of $\varepsilon_{\perp}$ are different in these regions [7]. The value of $\varepsilon_{\perp}$ is maximum in the centre of plasma and minimum in the space between the edge of plasma and the chamber wall, and these spaces can be considered as series capacitors.

The electrical equivalent circuit for tokamak after taking into account the capacitor property is shown in Figure 3 in which $V_{l}$ is the loop voltage, $R_{p}$ and $L$ are the resistance and inductance of the plasma which are calculated by $R_{P}=\left(0.002 \frac{R}{a_{1}^{2}}\right) Z_{\text {eff }} T_{e}^{-1 / 5}$ and $L=\mu_{0} R\left[\operatorname{Ln}\left(\frac{8 R}{a_{1}}\right)-\frac{7}{4}\right]$ relations, respectively. In these two equations, $Z_{\text {eff }}, T_{e}$ and $\mu_{0}$ are the effective charge of tokamak plasma, the electron temperature and the vacuum permeability coefficient, respectively [8] [9]. The equivalent circuit that has involved these capacitors consists of several meshes and its analytical solution is extremely difficult, but the results of a circuit with $n=2$ (Figure 3) are good enough for simulation the tokamak plasma. The circuit equation for Figure 3 is a second order differential equation in following form:

$$
L C \frac{\mathrm{d}^{2} i_{P}}{\mathrm{~d} t^{2}}+\left(G L+R_{P} C\right) \frac{\mathrm{d} i_{P}}{\mathrm{~d} t}+\left(G R_{P}+1\right) i_{P}=i_{S}(T)
$$

In Equation (8), $i_{s}$ is obtained using Norton equivalent circuit of Figure 3 in the following way:

$$
i_{s}(t)=\frac{\mathrm{e}^{-\left(R_{P} / L\right) t}}{L} \int_{0}^{t} \mathrm{e}^{\left(R_{P} / L\right) t^{\prime}} V_{l} \mathrm{~d} t^{\prime}
$$

The general solution of Equation (9) for the plasma current can be written as:

$$
i_{P}=\mathrm{e}^{-\alpha t}\left[I_{0} \frac{\omega_{0}}{\omega_{d}} \cos \left(\omega_{d} t-\varphi\right)+\frac{V_{0}}{L \omega_{d}} \sin \omega_{d} t+\frac{\omega_{0}^{2} \sin \omega_{d} t}{\omega_{d}} \int \mathrm{e}^{\alpha t} i_{s}(t) \cos \omega_{d} t \mathrm{~d} t-\frac{\omega_{0}^{2} \cos \omega_{d} t}{\omega_{d}} \int \mathrm{e}^{\alpha t} i_{s}(t) \sin \omega_{d} t \mathrm{~d} t\right]
$$



Figure 3. RLC equivalent circuit of tokamak plasmas. 
In Equation (10) $\omega_{0}=\sqrt{\frac{1+G R_{P}}{L C}}$ and $\alpha=\frac{\left(G L+R_{P} C\right)}{2 L C}$ are the natural frequency (resonance) and the damping constant, respectively. $V_{0}, I_{0}$ are the initial values of the plasma loop voltage and the plasma current, respectively. $\omega_{0}$ is calculated through $\omega_{d}=\sqrt{\omega_{0}^{2}-\alpha^{2}}$. Natural frequency $\omega_{0}=\sqrt{\frac{1+G R_{P}}{L C}} \cong \frac{1}{\sqrt{L C}}$ is an important factor that can play an important role in various regimes of the tokamak plasma. The values of natural frequencies for different tokamaks are in order of $\omega_{0} \approx 10^{5} \mathrm{rad} / \mathrm{s}$.

In fact, according to the properties of electrical elements, in dc regimes (following the end of transient regimes), $C$ would act such as an open circuit and $L$ such as a short cuircuit. Therefore, the circuit in Figure 3 is changed to a simple resistive circuit in which $V_{l}=R_{p} i_{p}$. When $V_{l}$ varies with time, not only the resistance circuit but also the $R L$ model cannot correctly simulate the behaviour of the plasma. Hence, $R L C$ model should be used.

The first effects of capacitance are weak damping oscillations with natural frequency in plasma current, radial electric fields and so on, detection of them is usually difficult. Its second effects are to cause some types of fluctuations in density and other plasma parameters at the edge of plasma. Such fluctuations have been observed in Damavand tokamak [4]. Probably, some fluctuations in $\mathrm{kHz}$ range in $\mathrm{H}$ mode of tokamak and in plasma edge can be related to the natural frequency discussed above. When the value of $V_{l}$ is constant no fluctuation would be seen and capacitive and inductive properties do not play an important role and the circuit in Figure 3 is changed to a simple resistive circuit.

\section{Results and Discussion}

The experimental data of the Damavand tokamak are [10]:

$$
n=3 \times 10^{19} \mathrm{~m}^{-3}, B_{T}=1.2 \mathrm{~T}, a_{1}=7 \mathrm{~cm}, R=36 \mathrm{~cm} .
$$

$I_{P}=40 \mathrm{kA}, T_{i}=150 \mathrm{eV}, T_{e}=300 \mathrm{eV}$ and the discharge time $t_{d}=15 \mathrm{~ms}$.

On the basis of dimension and condition of Damavand tokamak we would have:

$$
L \cong 0.9 \times 10^{-6} \mathrm{H}, \quad C \cong 1.7 \times 10^{-6} \mathrm{~F}, G \cong 0.01 \Omega^{-1} \text { and } \alpha \cong 10^{4} \text {. }
$$

After some calculating it would be: $\omega_{0} \cong 8 \times 10^{5} \mathrm{rad} / \mathrm{s} \gg \alpha$.

And, therefore, it would be: $\omega_{d} \cong \omega_{0} \cong 8 \times 10^{5} \mathrm{rad} / \mathrm{s}$ or $f \cong 1.2 \times 10^{5} \mathrm{~Hz}$.

As previously noted, the first effect of the capacitance is weak damping oscillations with the frequency $\omega_{d} \cong \omega_{0}$ that is the common result of an RLC circuit. However, when the loop voltage is dc, oscillation would not observe. Usually during the disruption instability a negative spike would observe in loop voltage. Such spikes in loop voltage and the condition of $\omega_{0} \gg \alpha$ in a tokamak leads to a solution for Equation (9) in the form of a weak damped oscillation $\mathrm{e}^{-\alpha t} \cos \omega_{d} t$.

In the experiments with Damavand tokamak that a sample of their results is demonstrated in Figure 4, when the negative spike of loop voltage is observed, the disruption instability happened and $\frac{\mathrm{d} I_{p}}{\mathrm{~d} t}$ showed weak damping oscillations. From the calculations using the presented model, it is seen that the frequency of these oscillations is approximately $100 \mathrm{kHz}$ with damping in form of $\mathrm{e}^{-10^{4} t}$. In Figure 4, the calculated signal by Equation (11) for an ideal shock input voltage is compared with experimental results and a good agreement between them is observed [10]. $\frac{\mathrm{d} I_{p}}{\mathrm{~d} t}$ is measured by a Rogowski coil.

When a power source (such as induced current, radio frequency wave, neutral beam) is injected into the plasma, there will be two working regimes:

1) The first one is a transient regime in which $L$ and $C$ along with $R$ play role in equation of circuit and to simulate the behaviour of the plasma, using $R L C$ circuit (Figure 3) with Equation (9) is necessary.

2) In the second regime that is a steady state regime, a simple resistive circuit with circuit equation of $V_{l}=R i_{p}$ is sufficient.

Therefore, the capacitance of the plasma like its inductance, plays important role in some working regimes of tokamak. The importance of capacitance properties is not limited to regimes with time-varying loop voltages. In 


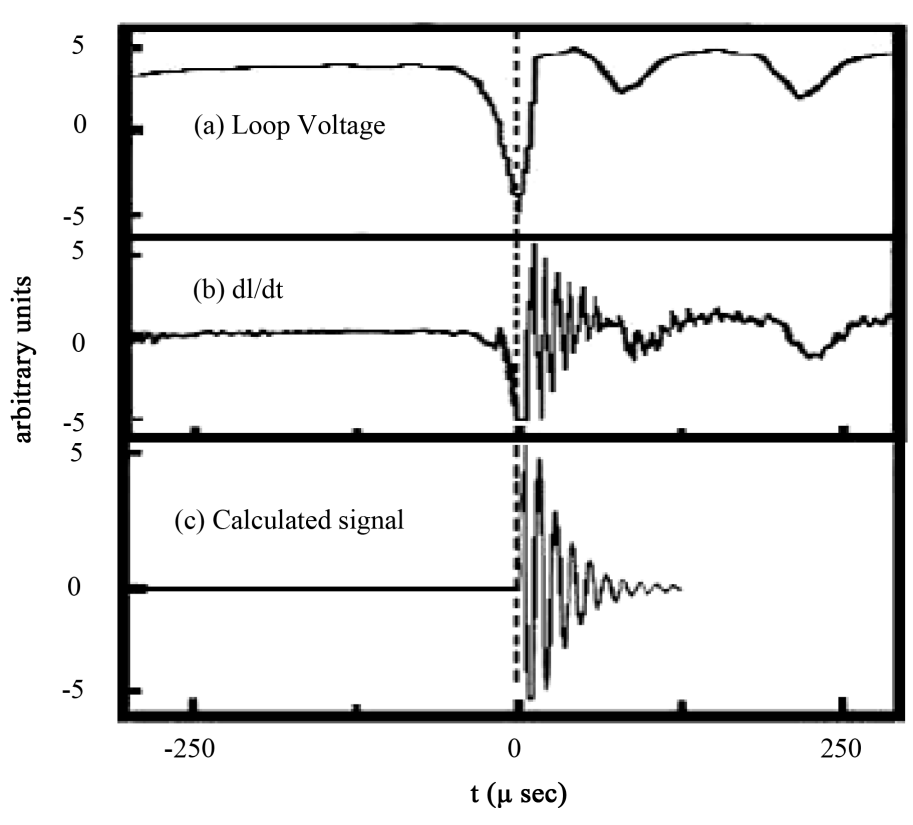

Figure 4. (a) The negative spike in loop voltage in Damavand tokamak; (b) weak damping oscillations $\frac{\mathrm{d} I}{\mathrm{~d} t}$ in Damavand tokamak; (c) Calculated signal $\frac{\mathrm{d} I}{\mathrm{~d} t}$ in Damavand tokamak using the $R L C$ model for this loop voltage.

each transient regime and regimes with time varying power source (such as radio frequency waves and neutral beam heating) the effect of capacitive property can be seen. In Figure 3, in addition to an electrical power supply, radio frequency waves and neutral beams can be considered as the power supply.

\section{Conclusions}

The radial electric field in study of the $H$ mode of tokamak plasmas is a very important parameter and several models have been proposed to explain the origin of them. In this paper, the effects of capacitive property on radial electric field in tokamak are briefly explained. It is proposed that a radial electric field is produced by a radial current in the form of $\varepsilon_{0} \varepsilon_{\perp} \frac{\partial}{\partial t} E_{r}=-J_{r}^{\text {tot }}$ [5] in which $J_{r}^{\text {tot }}$ is the total current in the radial direction.

Then, a new model for equivalent circuit of tokamak plasmas on the base of capacitive property is explained. The plasma has the capacitive property of $\varepsilon_{0} \varepsilon_{\perp}$ in all regions between its centre and the vacuum chamber wall. With respect to that value of $\varepsilon_{0} \varepsilon_{\perp}$ inside the plasma is maximum, the capacitance between centre of plasma and its edge is very high .Therefore, the capacitance in Equation (7) should be estimated as the capacitance of the space between plasma edge and the vaccum chamber that generates a radial electric field on the plasma edge in the form of $E_{r}=\frac{Q}{\left(a_{2}-a_{1}\right) C}$. We can investigate the generation of radial electric field in $H$ mode using this value for capacitance in Equation (8) and Figure 3 which gives more accurate results.

In this article, the analysis on the base of this model is first accomplished for Damavand tokamak and a good agreement between results of this model and the experimental results is observed. This model may be extended in future for analysis of the performance of big tokamaks such as ITER.

\section{References}

[1] Friedberg, J. (2007) Plasma Physics and Fusion Energy. 1st Edition, Cambridge University Press, Cambridge. http://dx.doi.org/10.1017/CBO9780511755705 
[2] Wesson, J. (1987) Tokamaks. 1st Edition, Oxford Science Publication, Oxford.

[3] Loarte, A. (2006) Chaos Cuts ELMs down to Size. Nature Physics, 2, 369-370. http://dx.doi.org/10.1038/nphys331

[4] Amrollahi, R. and Farshi, E. (1996) Modelling an RLC Circuit for the Investigation of the Disruption Instabilities in Tokamaks. Proceedings of the 16th IAEA Fusion Energy Conference, Montreal, 7-11 October 1996, 649-654.

[5] Itoh, K., Itoh, S.I. and Fukuyama, A. (1999) Transport and Structural Formation in Plasmas. 1st Edition, IOP Publishing, Bristol.

[6] Jackson, J.D. (1998) Classical Electrodynamics. 3rd Edition, John Wiley \& Sons, New York.

[7] Inan, U.S. and Gokowski, M. (2011) Principles of Plasma Physics for Engineers and Scientists. Cambridge University Press, Cambridge.

[8] Spitzer, L. and Harm, R. (1953) Transport Phenomena in a Completely Ionized Gas. Physical Review, 89, 977. http://dx.doi.org/10.1103/PhysRev.89.977

[9] Fish, N.J. (1985) Conductivity of RF-Heated Plasma. Physics of Fluids, 28, 245-247. http://dx.doi.org/10.1063/1.865186

[10] Farshi, E., Brevnov, N., Bortnikov, A., Gott, Y. and Shurygin, V. (2001) Some Characteristics of the Predisruption Phase in Tokamaks. Physic of Plasmas, 8, 3587-3594. http://dx.doi.org/10.1063/1.1379969 
Scientific Research Publishing (SCIRP) is one of the largest Open Access journal publishers. It is currently publishing more than 200 open access, online, peer-reviewed journals covering a wide range of academic disciplines. SCIRP serves the worldwide academic communities and contributes to the progress and application of science with its publication.

Other selected journals from SCIRP are listed as below. Submit your manuscript to us via either submit@scirp.org or Online Submission Portal.
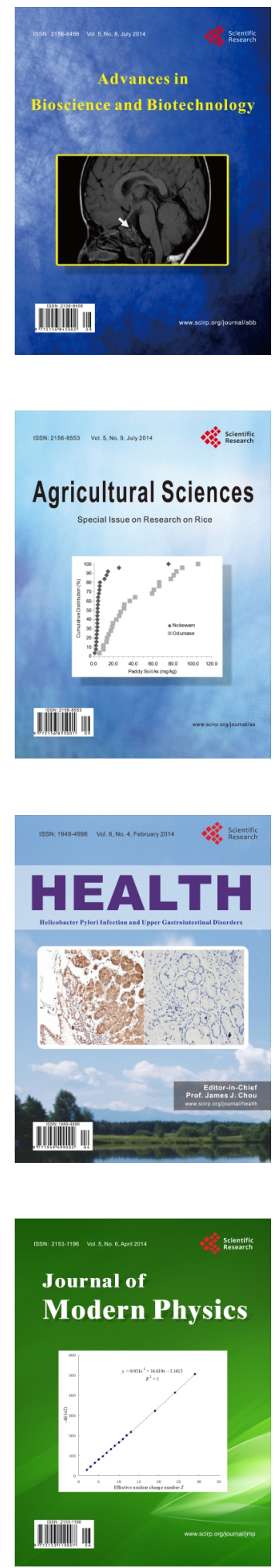
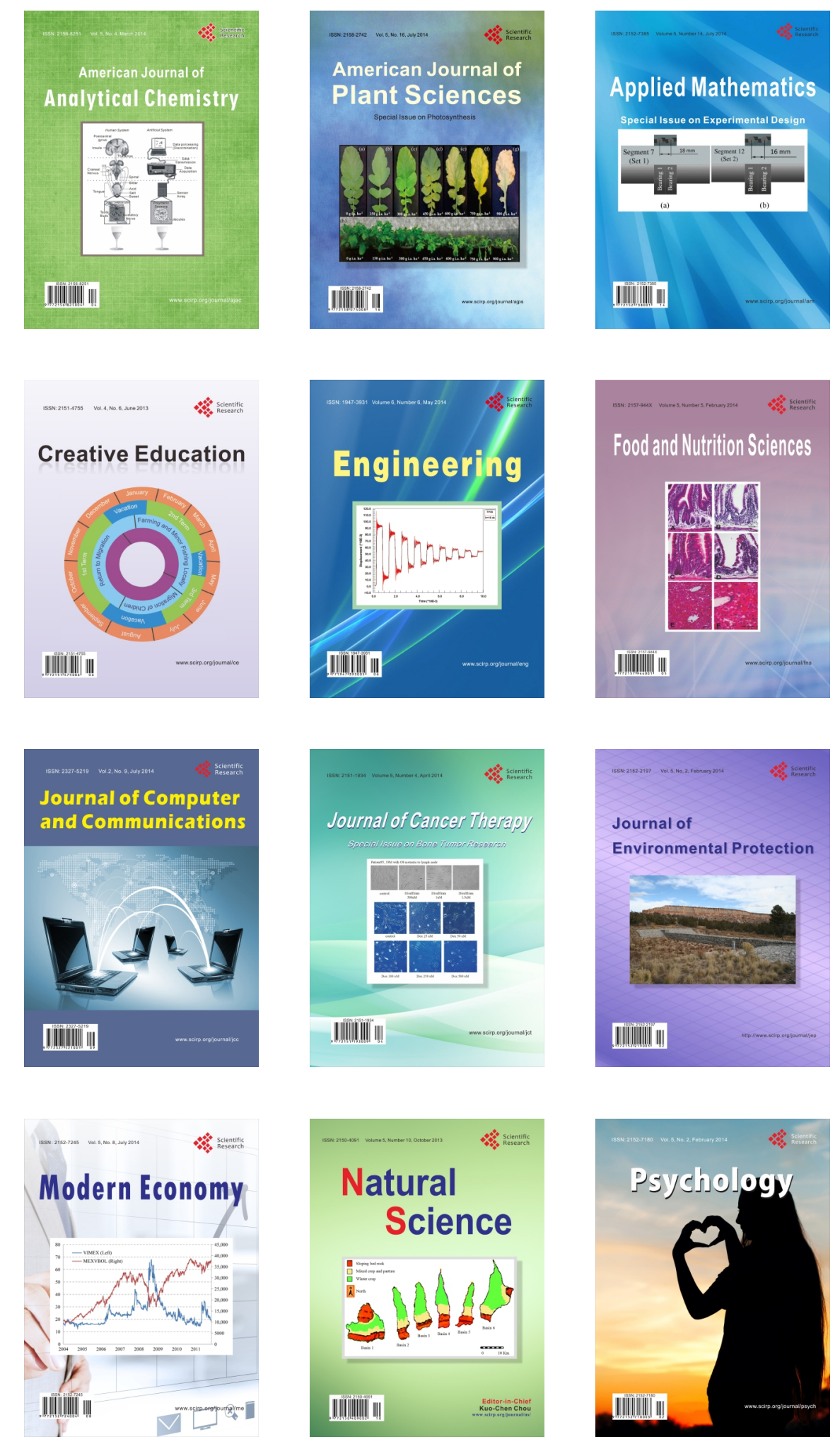